\title{
Влияние кристаллографической ориентации пленок GaSb на их структурные свойства при гетероэпитаксии на вицинальных подложках $\mathrm{Si}(001)$ методом молекулярно-лучевой эпитаксии
}

\author{
(C) М.О. Петрушков ${ }^{1}$, Д.С. Абрамкин ${ }^{1,2}$, Е.А. Емельянов ${ }^{1}$, М.А. Путято ${ }^{1}$, А.В. Васев ${ }^{1}$, И.Д. Лошкарев ${ }^{1}$ \\ М.Ю. Есин ${ }^{1}$, О.С. Комков ${ }^{3}$, Д.Д. Фирсов ${ }^{3}$, В.В. Преображенский ${ }^{1}$ \\ ${ }^{1}$ Институт фризики полупроводников им. А.В. Ржанова Сибирского отделения Российской академии наук, \\ 630090 Новосибирск, Россия \\ ${ }^{2}$ Новосибирский государственный университет, \\ 630090 Новосибирск, Россия \\ ${ }^{3}$ Санкт-Петербургский государственный электротехнический университет „ЛЭТИ“, \\ 197376 Санкт-Петербург, Россия \\ E-mail: maikdi@isp.nsc.ru
}

Поступила в Редакцию 3 августа 2020 г.

В окончательной редакции 10 августа 2020 г.

Принята к публикации 10 августа 2020 г.

\begin{abstract}
Методом молекулярно-лучевой эпитаксии с использованием переходных слоев $\mathrm{AlSb} / \mathrm{As} / \mathrm{Si}$ выращены пленки $\mathrm{GaSb}$ на вицинальных подложках $\mathrm{Si}(001)$, отклоненных на $6^{\circ}$ к плоскости (111). Исследовано влияние кристаллографической ориентации пленок $\mathrm{GaSb}$ на их структурные свойства и характер рельефа поверхности. Обнаружено, что пленки $\mathrm{GaSb}(00 \overline{1}) / \mathrm{Si}$ характеризуются лучшим структурным совершенством, меньшей концентрацией точечных дефектов и более планарным и изотропным рельефом поверхности по сравнению с пленками $\mathrm{GaSb}(001)$. Возможной причиной наблюдаемых отличий у пленок $\mathrm{GaSb}$ с различной ориентацией является повышенная плотность антифазных доменов в пленках $\mathrm{GaSb}(001)$. Морфологические особенности выращенных пленок обусловлены в основном наличием краев террас и в меньшей степени анизотропией встраивания адатомов $\mathrm{Ga}$ в края террас.
\end{abstract}

Ключевые слова: молекулярно-лучевая эпитаксия, $\mathrm{GaSb}$ на $\mathrm{Si}(001)$, кристаллографическая ориентация пленки, структурное совершенство, морфология поверхности.

DOI: 10.21883/FTP.2020.12.50227.9496

\section{1. Введение}

В последнее время уделяется большое внимание созданию различных оптоэлектронных приборов ИК диапазона на основе $\mathrm{A}^{\mathrm{III}} \mathrm{Sb}$, таких как лазеры, светодиоды, фотодиоды и др. [1-3]. Структуры для устройств на основе $\mathrm{A}^{\mathrm{III}} \mathrm{Sb}$ обычно выращивают на дорогих подложках $\mathrm{GaSb}$, которые к тому же характеризуются низкой механической прочностью. В связи с этим актуальной задачей является разработка технологии получения тонких высококачественных слоев $\mathrm{GaSb}$ на дешевых и прочных подложках $\mathrm{Si}$. Применение эпитаксиальных искусственных подложек $\mathrm{GaSb} / \mathrm{Si}$ позволит значительно снизить стоимость оптоэлектронных приборов на основе $\mathrm{A}^{\mathrm{III}} \mathrm{Sb}$ и обеспечить им широкий круг применений. Кроме того, интеграция оптоэлектронных устройств ближнего и среднего ИК диапазона, основанных на $\mathrm{GaSb}$, и кремниевой электроники открывает перспективу существенного ускорения обработки информации за счет передачи данных по оптическому каналу [4].

При гетероэпитаксии соединений $\mathrm{A}^{\mathrm{III}} \mathrm{B}^{\mathrm{V}}$ на кремнии в объеме пленки образуются как точечные, так и протяженные структурные дефекты, снижающие эксплуатационные свойства искусственных $\mathrm{A}^{\mathrm{III}} \mathrm{B}^{\mathrm{V}} / \mathrm{Si}$ подложек. Основными протяженными структурными дефектами яв- ляются дислокации несоответствия (ДН) и антифазные границы (АФГ). Причиной возникновения ДН является релаксация механических напряжений [5], вызванных различием в значениях постоянных решеток и температурных коэффициентов линейного расширения кремния и материалов $\mathrm{A}^{\mathrm{III}} \mathrm{B}^{\mathrm{V}}$. Возникновение АФГ обусловлено сбоем чередования слоев катионной и анионной подрешеток кристалла при зарождении эпитаксиального слоя полярного соединения $\mathrm{A}^{\mathrm{III}} \mathrm{B}^{\mathrm{V}}$ на неполярной подложке $\mathrm{Si}$ [6]. На границах таких областей (так называемых антифазных доменов (АФД)) имеет место повышенная концентрация точечных дефектов, которые могут выступать в роли центров безызлучательной рекомбинации и негативно влияют на оптические свойства получаемых гетероструктур $\mathrm{A}^{\mathrm{III}} \mathrm{B}^{\mathrm{V}} / \mathrm{Si}$ [7]. Кроме того, наличие ДН и АФГ может приводить к огрублению поверхности растущей пленки $\mathrm{A}^{\mathrm{III}} \mathrm{B}^{\mathrm{V}} / \mathrm{Si}$, что также негативно сказывается на возможности приборного применения получаемых искусственных подложек.

В силу большого рассогласования постоянных решеток $\mathrm{GaSb}$ и $\mathrm{Si}, \sim 12 \%$, процесс образования сетки ДН начинается при формировании уже первых монослоев $\mathrm{GaSb}$. АФД также образуются в процессе зарождения первых монослоев (МC) эпитаксиальной пленки $\mathrm{A}^{\mathrm{III}} \mathrm{B}^{\mathrm{V}}$. Поэтому актуальными являются исследования, направленные на выявление закономерностей, связывающих 
условия зарождения со структурными свойствами и морфологией поверхности пленок $\mathrm{GaSb} / \mathrm{Si}$.

Для подавления процесса формирования АФД в пленках $\mathrm{A}^{\mathrm{III}} \mathrm{B}^{\mathrm{V}} / \mathrm{Si}$ применяют вицинальные подложки ориентации (001), отклоненные на 4-6 ${ }^{\circ}$ к плоскости (111). В процессе отжига на поверхности таких подложек формируется система двухатомных ступеней $[8,9]$, которая способствует согласованию порядка следования атомов в участках пленки $\mathrm{A}^{\mathrm{III}} \mathrm{B}^{\mathrm{V}}$, зарождающихся на различных террасах Si-подложки. Использование неполярной вицинальной подложки кремния для выращивания полярного материала позволяет формировать пленки $\mathrm{A}^{\mathrm{III}} \mathrm{B}^{\mathrm{V}} / \mathrm{Si}$ c различной ориентацией - $\mathrm{A}^{\mathrm{III}} \mathrm{B}^{\mathrm{V}}(001) / \mathrm{Si}(001)$ и $\mathrm{A}^{\mathrm{III}} \mathrm{B}^{\mathrm{V}}(00 \overline{1}) / \mathrm{Si}(001)[10,11]$. Эти пленки отличаются ориентацией димеров, образующих сверхструктуру поверхности (001), относительно краев вицинальных террас. Стоит отметить, что сингулярные поверхности (001) и $(00 \overline{1})$ соединений $\mathrm{A}^{\mathrm{III}} \mathrm{B}^{\mathrm{V}}$ не различимы с точки зрения атомного строения и физико-химических свойств. В то же время, как было показано в работах $[10,12]$, при росте GaAs на вицинальных подложках $\mathrm{Si}$, ориентация димеров мышьяка относительно краев террас существенно влияет на рельеф эпитаксиальных пленок. Вопрос о влиянии ориентации димеров на свойства пленок $\mathrm{GaSb} / \mathrm{Si}(001)$ в литературе практически не обсуждался.

Настоящая работа посвящена исследованию влияния кристаллографической ориентации пленок $\mathrm{GaSb}$ (001) или (001), выращенных на вицинальных подложках $\mathrm{Si}(001)$ методом молекулярно-лучевой эпитаксии (МЛЭ), на их структурные свойства и морфологию поверхности.

\section{2. Методика эксперимента}

Гетероструктуры $\mathrm{GaSb} / \mathrm{Si}$ выращивались на установке МЛЭ „Штат“. Для получения потоков $\mathrm{Ga}, \mathrm{Sb}$ и $\mathrm{Si}$ использовались тигельные молекулярные источники, а для получения потока молекул $\mathrm{As}_{2}$ использовался вентильный источник с зоной крекинга [13]. Для подавления образования в эпитаксиальных пленках АФД использовались подложки $\mathrm{Si}(001)$, отклоненные на $6^{\circ}$ к плоскости (111) $[8,9]$. Контроль за структурным состоянием и морфологией поверхности во время роста осуществлялся методом дифракции быстрых электронов на отражение (ДБЭО).

Удаление окисного слоя с поверхности подложки $\mathrm{Si}$ проводилось при температуре подложки $\left(T_{S}\right) 750^{\circ} \mathrm{C}$ в потоке атомарного кремния (поток соответствовал скорости роста 0.01 атомных слоев в секунду). После удаления оксида, подложка отжигалась при $T_{S}=850^{\circ} \mathrm{C}$ в течение 30 мин для формирования системы двухатомных террас на поверхности Si. Согласно данным работы [11], димерные связи $\mathrm{Si}-\mathrm{Si}$ на такой поверхности ориентированы вдоль краев террас, при этом сверхструктурное состояние поверхности характеризуется симметрией $(2 \times 1)$. После отжига подложка охлаждалась в условиях сверхвысокого вакуума до температуры формирования переходного слоя.
Переходный слой состоял из двух подслоев: слоя зарождения и слоя планаризации. Слой зарождения служит основой для эпитаксии соединений $\mathrm{A}^{\mathrm{III}} \mathrm{B}^{\mathrm{V}}$ на поверхности кремния. В данной работе слой зарождения представлял собой димеризированный слой атомов мышьяка. От условий формирования этого слоя зависит кристаллографическая ориентация последующих слоев соединений $\mathrm{A}^{\mathrm{III}} \mathrm{B}^{\mathrm{V}}$. Как показано в работе [11], в зависимости от условий осаждения атомы Аs хемосорбируются либо с замещением, либо без замещения верхнего слоя атомов Si. При этом димерные связи As-As оказываются расположенными либо параллельно, либо перпендикулярно краям террас, и при последующем росте материала $\mathrm{A}^{\mathrm{III}} \mathrm{B}^{\mathrm{V}}$ формируются слои с ориентацией $(001)$ и $(00 \overline{1})$ соответственно. Введем обозначение слоя димеров мышьяка, покрывающего поверхность кремния: „As $(00 \overline{1})^{\text {“ }}$, для которого связи в димерах As-As расположены перпендикулярно краям террас, и „As $(001)^{“}$, для которого связи As-As параллельны краям террас. Температура подложки при формировании слоев $\mathrm{As}(00 \overline{1})$ и $\mathrm{As}(001)$ равнялась 200 и $450^{\circ} \mathrm{C}$ соответственно.

Слой планаризации выращивается с целью формирования сплошной тонкой пленки соединений $\mathrm{A}^{\mathrm{III}} \mathrm{B}^{\mathrm{V}}$ с как можно более гладкой поверхностью. В соответствии с результатами работ $[14,15]$ использование слоев $\mathrm{AlSb}$ перед ростом $\mathrm{GaSb}$ на $\mathrm{Si}$-подложках способствует формированию тонкого сплошного слоя $\mathrm{GaSb}$ с планарной поверхностью. В нашей работе в качестве слоя планаризации использовался слой $\mathrm{AlSb}$ номинальной толщиной $10 \mathrm{MC}$, выращенный при температуре $350^{\circ} \mathrm{C}$ методом атомно-слоевой эпитаксии (АСЭ), заключающимся в поочередном осаждении атомарных слоев Al и Sb. Таким образом, были сформированы переходные слои $\operatorname{AlSb}(00 \overline{1}) / \mathrm{Al} / \mathrm{As}(00 \overline{1}) / \mathrm{Si}$ и $\mathrm{AlSb}(001) / \mathrm{Al} / \mathrm{As}(001) / \mathrm{Si}$, позволяющие выращивать пленки $\mathrm{GaSb}(00 \overline{1})$ и $\mathrm{GaSb}(001)$ соответственно.

На переходных слоях выполнялся рост слоев $\mathrm{GaSb}$ номинальной толщиной $5 \mathrm{MC}$ методом АСЭ. Основные слои $\mathrm{GaSb}$ толщиной 500 нм выращивались методом МЛЭ со скоростью $1 \mathrm{MC} / \mathrm{c}$ при $T_{S}=400^{\circ} \mathrm{C}$. Относительно низкая температура роста, по сравнению с используемой в работах [14-17] $\left(500-600^{\circ} \mathrm{C}\right)$, была выбрана с целью снижения влияния $T_{S}$ на процессы изменения состояния комплекса протяженных и точечных дефектов, сформированных на начальных этапах роста. Такой подход позволил более явно проявиться влиянию условий зарождения переходных слоев на свойства эпитаксиальных пленок $\mathrm{GaSb} / \mathrm{Si}$.

После роста структуры подвергались циклическому отжигу. Цикл состоял из нагрева от 250 до $520^{\circ} \mathrm{C}$ в течение 40 с, выдержки при $520^{\circ} \mathrm{C}$ в течение 30 с и естественного охлаждения до $250^{\circ} \mathrm{C}$ в течение 2.5 мин. Всего было проведено по 5 циклов для каждой структуры.

Для сравнения была выращена гомоэпитаксиальная структура $\mathrm{GaSb} / \mathrm{GaSb}$ толщиной 1 мкм. Структура выращивалась в установке RIBER-32P на $p^{+}-\mathrm{GaSb}$-подложке, легированной германием. 

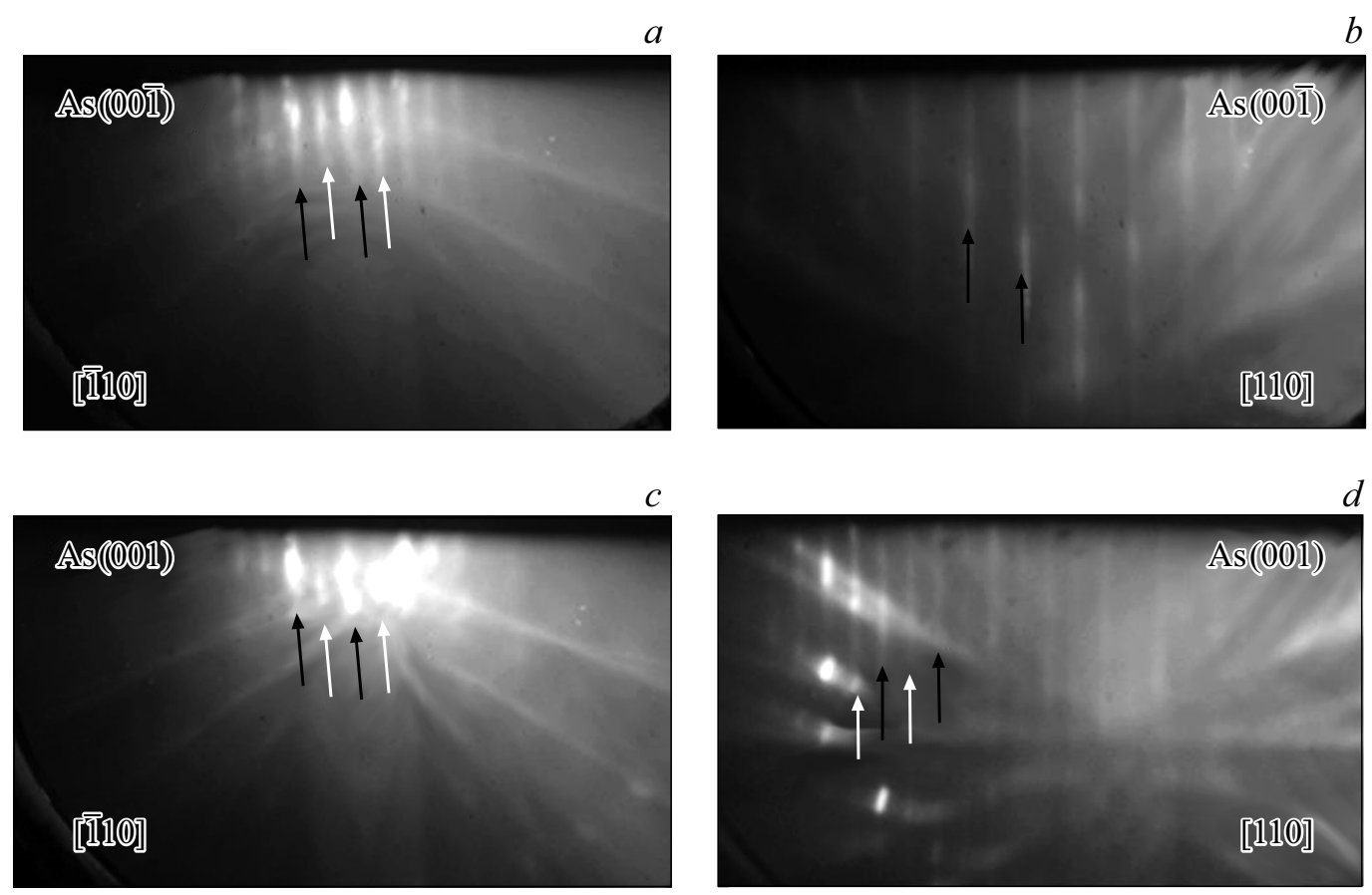

Рис. 1. Картина ДБЭО от слоев: $a-\operatorname{As}(00 \overline{1}) / \mathrm{Si}$ (азимут $[\overline{1} 10]), b-\operatorname{As}(00 \overline{1}) / \mathrm{Si}($ азимут $[110]), c-\operatorname{As}(001) / \mathrm{Si}($ азимут $[\overline{1} 10])$, $d-\mathrm{As}(001) / \mathrm{Si}$ (азимут [110]). Белыми стрелками на рисунках указаны дробные рефлексы $1 / 2$, черными стрелками - основные рефлексы.

Морфология поверхности выращенных пленок исследовалась методом атомно-силовой микроскопии (АСM) на микроскопе Solver P47 (NT-MDT) в полуконтактном режиме на образцах без отжига (as grown). Кристаллические свойства образцов исследованы методом рентгеновской дифрактометрии (РД), а оптические методом спектроскопии фотолюминесценции (ФЛ). Измерения кривых качания РД проведены с помощью дифрактометра DSO-1T с использованием кристалламонохроматора $\mathrm{Ge}(004)$ в излучении $\mathrm{Cu} K_{\alpha 1}$. Спектры ФЛ измерялись при помощи установки на базе инфракрасного фурье-спектрометра Vertex 80, оснащенного $\mathrm{KBr}$-светоделителем и InSb-фотодетектором, охлаждаемым жидким азотом. Для возбуждения люминесценции был задействован 809 нм лазерный диод, излучение которого фокусировалось на поверхности образцов в пятно площадью $\sim 2 \mathrm{Mм}^{2}$. При этом плотность мощности возбуждения ФЛ составляла $\sim 30 \mathrm{BT} / \mathrm{cm}^{2}$. Образцы размещались в гелиевом криостате замкнутого цикла Janis CCS-150. Температура на держателе образцов составляла $10 \mathrm{~K}$. Фурье-спектрометр был модифицирован для вывода сигнала с предусилителя фотоприемника на синхронный усилитель SR-830. Для проведения измерений была задействована методика с синхронным усилением сигнала фотолюминесценции, описанная в работе [18]. Используемый подход позволяет одновременно добиться усиления полезного сигнала ФЛ и исключить из спектра нежелательный фон теплового излучения, достаточно интенсивный в среднем ИК диапазоне.

\section{3. Экспериментальные результаты}

Картины ДБЭО, полученные при осаждении слоя $\operatorname{As}(00 \overline{1}) / \mathrm{Si}$, снятые в азимутах [110] (вдоль краев террас) и [110] (поперек), представлены на рис. $1, a$ и $b$ соответственно. При наблюдении в азимуте $[\overline{1} 10]$ (рис. $1, a$ ) видны как основные, так и дробные $1 / 2$ рефлексы, сформированные при дифракции электронов на упорядоченных димерных рядах, ориентированных вдоль краев террас. Основные рефлексы отмечены на рисунке черными стрелками, дробные - белыми. При наблюдении в направлении поперек террас, в азимуте [110], дробные рефлексы не различимы (рис. $1, b)$. Таким образом, на поверхности $\operatorname{As}(00 \overline{1}) / \mathrm{Si}$ имеет место поверхностная структура $(1 \times 2)$. На рис. $1, a$ видно расщепление основных рефлексов, обусловленное дифракцией от упорядоченной системы двухатомных террас. Наклон (относительно краевой линии) рефлексов на рис. 1, $a$ и $c$ обусловлен отклонением подложки от сингулярной грани (001).

Картины ДБЭО от слоя $\mathrm{As}(001) / \mathrm{Si}$, снятые в различных азимутах, представлены на рис. $1, c$ и $d$. В обоих азимутах видны как основные, так и дробные $1 / 2$ рефлексы, обозначенные на рисунке черными и белыми стрелками соответственно. На рис. 1, с укороченные дробные рефлексы 1/2 связаны с дифракцией электронов на участках с рядами димеров мышьяка, ориентированными вдоль краев террас. На рис. $1, d$ наблюдаются узкие и длинные дробные $1 / 2$ рефлексы, связанные с дифракцией на димерных рядах, ориентированных поперек террас. Таким образом, на картинах ДБЭО наблюдается 


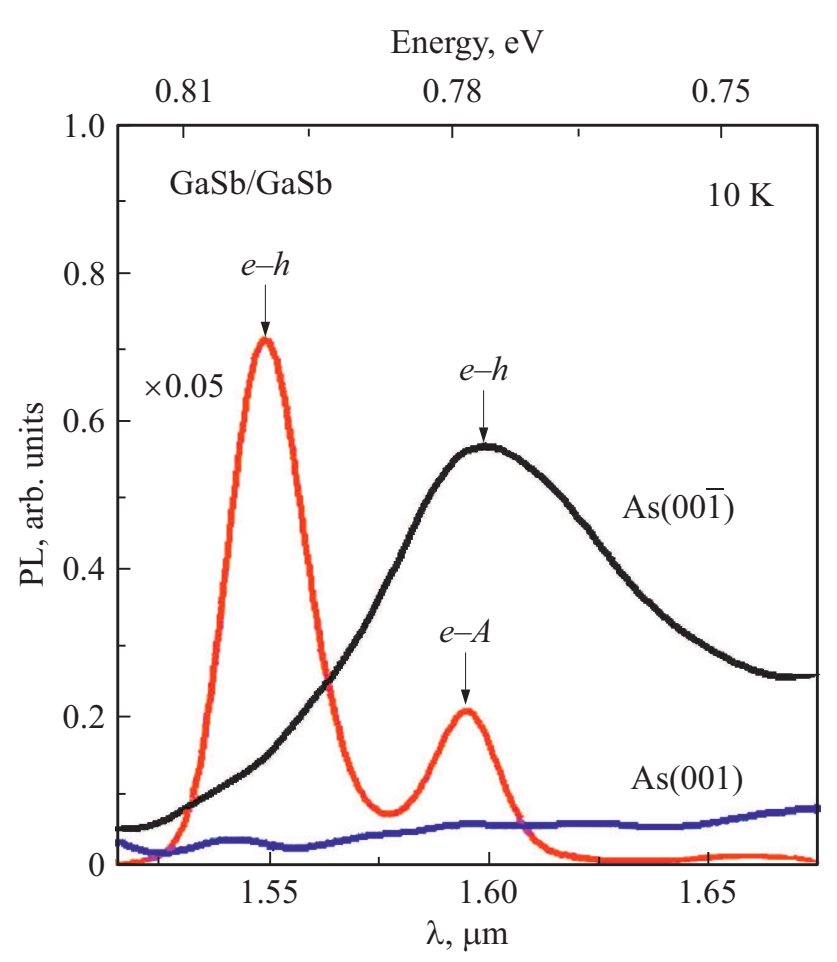

Рис. 2. Спектры стационарной ФЛ структур $\mathrm{GaSb} / \mathrm{Si}$, измеренные при температуре $10 \mathrm{~K}$. Спектры структур с ориентацией (001) и (001) отмечены на рисунке как $\operatorname{As}(001)$ и $\operatorname{As}(00 \overline{1})$ соответственно. Также приведен спектр структуры GaSb/GaSb.

смешанная реконструкция $(2 \times 2)$ от поверхности, на которой представлены оба типа доменов. Более четкие дробные рефлексы $1 / 2$ в азимуте [110] свидетельствуют о том, что преобладает фаза $\mathrm{As}(001) / \mathrm{Si}$.

По данным рентгеноструктурного анализа, структура $\mathrm{GaSb}(00 \overline{1}) / \mathrm{Si}$ характеризуется меньшим значением ши-
Результаты анализа данных АСМ для образцов $\mathrm{GaSb} / \mathrm{Si}$

\begin{tabular}{c|c|c|c|c|c}
\hline Образец & $L_{[\overline{1} 10]}$, нм & $L_{[110]}$, нм & $H$, нм & $A$ & $S$, мкм $^{2}$ \\
\hline $\mathrm{GaSb}(00 \overline{1})$ & 233.8 & 152.6 & 2.7 & 1.53 & 0.036 \\
$\mathrm{GaSb}(001)$ & 168.6 & 93.6 & 5.6 & 1.8 & 0.016
\end{tabular}

рины пика кривой качания (ПШПВ) $\left(670^{\prime \prime}\right)$ по сравнению со структурой с ориентацией (001) $\left(885^{\prime \prime}\right)$.

Спектры ФЛ структур $\mathrm{GaSb}(001) / \mathrm{Si}, \mathrm{GaSb}(00 \overline{1}) / \mathrm{Si}$ и $\mathrm{GaSb} / \mathrm{GaSb}$, измеренные при температуре $10 \mathrm{~K}$, представлены на рис. 2. В спектрах ФЛ структуры $\mathrm{GaSb} / \mathrm{GaSb}$ доминирует полоса с максимумом при энергии 800 мэВ и шириной 10 мэВ, связанная с межзонной рекомбинацией носителей заряда в $\mathrm{GaSb}$, а также полоса с максимумом на энергии 777 мэВ и шириной 9 мэВ, связанная с рекомбинацией свободных электронов и дырок, локализованных на акцепторах [19]. В спектрах гетероструктуры $\mathrm{GaSb}(00 \overline{1}) / \mathrm{Si}$ присутствует полоса ФЛ с максимумом на энергии 775 мэВ и шириной 26 мэВ, которую мы связываем с межзонной рекомбинацией в GaSb. Полоса межзонной рекомбинации для гетероструктуры $\mathrm{GaSb}(00 \overline{1}) / \mathrm{Si}$ характеризуется в $\sim 25$ раз меньшей интенсивностью, по сравнению с ФЛ структуры $\mathrm{GaSb} / \mathrm{GaSb}$, и смещена в низкоэнергетическую часть спектра на 25 мэВ относительно ФЛ $\mathrm{GaSb} / \mathrm{GaSb}$. Как мы считаем, спектральный сдвиг обусловлен наличием остаточных деформаций растяжения слоев GaSb. Pacчеты, проведенные в рамках приближения сплошной среды [20], позволяют оценить величину деформаций как $0.37 \%$. Ширина полос межзонной рекомбинации структур $\mathrm{GaSb} / \mathrm{GaSb}$ и $\mathrm{GaSb}(00 \overline{1}) / \mathrm{Si}$ составила 10 и $26 \mathrm{мэB}$ соответственно. Сигнал ФЛ от структуры $\mathrm{GaSb}(001) / \mathrm{Si}$ в диапазоне длин волн, соответствующем межзонным переходам, не превосходит уровень шума.
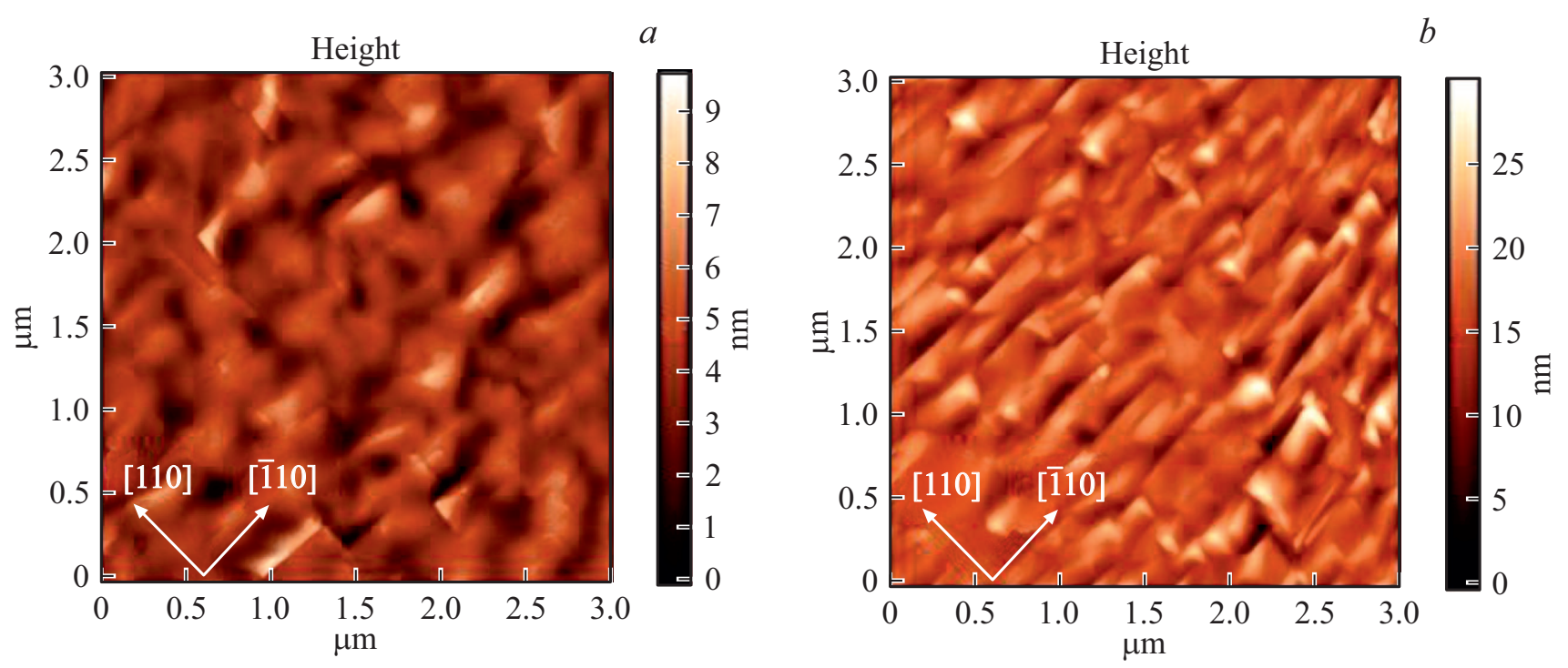

Рис. 3. АСМ изображения ростовой поверхности пленок: $a-\mathrm{GaSb}(00 \overline{1}) / \mathrm{Si}, b-\mathrm{GaSb}(001) / \mathrm{Si}$. 
На рис. 3 представлены АСМ изображения поверхности пленок $\mathrm{GaSb} / \mathrm{Si}$ с разной ориентацией. В левом нижнем углу для каждого изображения указана система координат, связанная с основными кристаллографическими направлениями подложки. Ориентация образцов относительно этих направлений осуществлялась методом ДБЭО. Морфология поверхности пленок на рис. 3 имеет явно выраженную анизотропию. Особенности рельефа вытянуты вдоль направления [110] (параллельно краям террас). При обработке АCM данных были построены гистограммы и 2D автокорреляционные функции. Проведенный анализ позволил определить вертикальные $(H)$ и латеральные $\left(L_{[\overline{1} 10]}\right.$ и $\left.L_{[110]}\right)$ размеры основных элементов рельефа, их площадь $\left(S=L_{[\overline{1} 10]} \times L_{[110]}\right)$ и степень анизотропии $\left(A=L_{[\overline{1} 10]} / L_{[110]}\right)$ (см. таблицу).

\section{4. Обсуждение результатов}

Как показывают наши экспериментальные данные, структура $\mathrm{GaSb}(00 \overline{1}) / \mathrm{Si}$ характеризуется меньшим значением ПШПВ кривых качания РД, существенно более высокой интенсивностью межзонной ФЛ и более планарным и изотропным рельефом поверхности по сравнению со структурой $\mathrm{GaSb}(001) / \mathrm{Si}$. Это свидетельствует о том, что структура $\mathrm{GaSb}(00 \overline{1}) / \mathrm{Si}$ обладает более высоким кристаллическим совершенством объема, меньшей концентрацией центров безызлучательной рекомбинации и более гладкой поверхностью соответственно. Следовательно, для получения качественных эпитаксиальных искусственных подложек $\mathrm{GaSb} / \mathrm{Si}$, пригодных для приборного применения, предпочтительными являются условия, в которых формируется пленка GaSb c ориентацией $(00 \overline{1})$. Далее мы обсудим возможные причины отличий свойств пленок $\mathrm{GaSb} / \mathrm{Si}$ с различной ориентацией.

Рассмотрим процесс формирования слоя зарождения $\operatorname{As}(00 \overline{1}) / \mathrm{Si}$. Он происходит путем хемосорбции молекул мышьяка на поверхности кремния при $T_{S}=100-200^{\circ} \mathrm{C}$. Из-за низкой температуры подложки процесс замещения атомов кремния атомами мышьяка сильно подавлен, следовательно, слой атомов мышьяка создает реплику поверхности Si-подложки. Структура мышьяковой реплики отличается от структуры подложки ориентацией димерных связей: в то время как поверхностные димеры $\mathrm{Si}-\mathrm{Si}$ на двухатомных террасах ориентированы вдоль краев террас, димеры As-As ориентируются перпендикулярно краям террас. Такой поверхности соответствует реконструкция $(1 \times 2)$, что и показывают наши данные ДБЭО. Данные об упорядоченности структуры поверхности $\mathrm{As}(00 \overline{1}) / \mathrm{Si}$ согласуются с данными сканирующей туннельной микроскопии (СТМ), представленными в работе [11].

В ходе экспериментов по выращиванию структуры $\mathrm{GaSb}(001) / \mathrm{Si}$ использовался слой зарождения $\mathrm{As}(001) / \mathrm{Si}$, сформированный при более высокой темпеpaтуре $T_{S}=450^{\circ} \mathrm{C}$. При этой температуре преимущественным становится уже процесс замещения атомов
$\mathrm{Si}$ атомами мышьяка. При замещении атомов $\mathrm{Si}$ на атомы As происходит формирование слоя $\mathrm{As}(001) / \mathrm{Si}$, в котором поверхностные димеры As-As ориентированы параллельно краям террас. Поскольку эти два процесса (хемосорбции с замещением и без замещения) реализуются параллельно, то существует вероятность формирования двухдоменной поверхности [11]. В условиях замещения атомов $\mathrm{Si}$ атомами мышьяка формирование двухдоменной поверхности проявляется на картинах ДБЭО в виде реконструкции $(2 \times 2)$ (см. рис. $1, c$ и $d)$.

Смешанный характер слоя зарождения $\mathrm{As} / \mathrm{Si}$ в структуре $\mathrm{GaSb}(001) / \mathrm{Si}$ позволяет сделать предположение о повышенной плотности АФД в такой структуре по сравнению со структурой $\mathrm{GaSb}(00 \overline{1}) / \mathrm{Si}$. Это обстоятельство оказывает существенное влияние на структурные свойства объема пленки $\mathrm{GaSb} / \mathrm{Si}$.

На границе АФД в $\mathrm{GaSb}$ будут локализованы связи $\mathrm{Ga}-\mathrm{Ga}$ и $\mathrm{Sb}-\mathrm{Sb}$. C одной стороны, это приводит к формированию глубоких уровней в запрещенной зоне $\mathrm{GaSb}$, выступающих как центры безызлучательной рекомбинации [21]. Это должно снижать эффективность излучательной межзонной рекомбинации носителей заряда в структурах $\mathrm{GaSb}(001) / \mathrm{Si}$ по сравнению с $\mathrm{GaSb}(00 \overline{1}) / \mathrm{Si}$, что и демонстрируют наши данные ФЛ. С другой стороны, из-за отличия в геометрии и энергии этих связей по сравнению со связями $\mathrm{Ga}-\mathrm{Sb}$ будет происходить деформация решетки, что должно оказывать влияние на формирование и движение дислокаций. Так, в работе [22] было показано, что в системе GaAs/Si возможно декорирование антифазных доменов дислокациями. Наличие АФД может затруднять движение ветвей пронизывающих дислокаций, поэтому в процессе роста снижение их плотности будет протекать менее эффективно. Чем больше протяженность антифазных границ, тем выше будет плотность пронизывающих ветвей дислокаций в выращенной пленке, что проводит к уширению кривых качания РД, что также отражается в наших экспериментальных данных.

Что касается морфологии, то заметным отличием является наличие более выраженной анизотропии рельефа для пленки $\mathrm{GaSb}(001)$ по сравнению с пленкой $\mathrm{GaSb}(00 \overline{1})$.

Структура рельефа поверхности определяется особенностями поверхностной диффузии и особенностями встраивания адатомов в процессе роста. С одной стороны, при росте на вицинальной подложке диффузия адатомов в направлении поперек краев террас затруднена из-за наличия барьера Швебеля [23,24]. С другой стороны, на поверхностную диффузию адатомов и вероятность встраивания оказывают существенное влияние особенности реконструкции поверхности $[12,25,26]$. По данным работы [26], эффективность встраивания адатомов $\mathrm{Ga}$ в ступень с ориентацией [110] выше по сравнению с эффективностью встраивания в ступень с ориентацией $[\overline{1} 10]$. Это является причиной анизотропии роста островков и при определенных условиях приводит к возникновению анизотропии рельефа поверхности. 
В нашем случае для пленок $\mathrm{GaSb}(001)$ и $\mathrm{GaSb}(00 \overline{1})$ особенности рельефа вытянуты вдоль краев террас, направление которых совпадает с направлением [1110] подложки. В случае $\mathrm{GaSb}(001)$ это направление совпадает с направлением эффективного встраивания адатомов галлия, а в случае $\mathrm{GaSb}(00 \overline{1})$ - перпендикулярно ему. Таким образом, наши результаты позволяют заключить, что при формировании рельефа пленок $\mathrm{GaSb} / \mathrm{Si}$ в условиях сильного отклонения $\left(6^{\circ}\right)$ доминирующим фактором можно считать наличие краев террас, что согласуется с результатами работы [12].

Кроме того, поверхности пленок $\mathrm{GaSb}(00 \overline{1}) / \mathrm{Si}$ характеризуются заметно меньшим вертикальным размером рельефа по сравнению с $\mathrm{GaSb}(001) / \mathrm{Si}$. Это может объясняться как особенностями встраивания и поверхностной диффузией адатомов, обсуждаемыми выше, так и большей плотностью АФД и ПД в структурах с ориентацией (001).

\section{5. Заключение}

Выращены структуры $\mathrm{GaSb}(001) / \mathrm{Si}$ и $\mathrm{GaSb}(00 \overline{1}) / \mathrm{Si}$ на подложках, отклоненных на $6^{\circ}$ к плоскости (111). Структуры формировались методом молекулярно-лучевой эпитаксии на переходных слоях $\mathrm{AlSb} / \mathrm{As} / \mathrm{Si}$. Ориентация пленок $\mathrm{GaSb}$ задавалась ориентацией димеров мышьяка относительно краев террас вицинальной подложки путем выбора условий зарождения слоя $\mathrm{As} / \mathrm{Si}$. Исследовано влияние кристаллографической ориентации пленок $\mathrm{GaSb}$ на их структурное совершенство, морфологию и оптические свойства.

Установлено, что пленки $\mathrm{GaSb}(00 \overline{1}) / \mathrm{Si}$ характеризуются меньшей шириной пика рентгеновских кривых качания, большей интенсивностью межзонной ФЛ и более планарным и изотропным рельефом поверхности по сравнению с пленками $\mathrm{GaSb}(001) / \mathrm{Si}$. Возможной причиной наблюдаемых отличий является повышенная плотность АФД в пленках с ориентацией (001), вызванная двухдоменностью структуры $\mathrm{GaSb}$ из-за особенностей формирования слоя зарождения $\mathrm{As} / \mathrm{Si}$.

Морфологические особенности выращенных пленок обусловлены в основном наличием краев террас и в меньшей степени анизотропией встраивания адатомов галлия в края террас.

\section{Благодарности}

Авторы выражают благодарность В.А. Соловьёву за выращивание структуры $\mathrm{GaSb} / \mathrm{GaSb}$.

\section{Финансирование работы}

Работа выполнена при поддержке государственного задания 0306-2020-0011.

\section{Конфликт интересов}

Авторы заявляют, что у них нет конфликта интересов.

\section{Список литературы}

[1] A. Joullie, P. Christol. C.R. Physique. 4 (6), 621 (2003).

[2] M. Razeghi, A. Haddadi, A.M. Hoang, E.K. Huang, G. Chen, S. Bogdanov, S.R. Darvish, F. Callewaert, R. McClintock. Infr. Phys. Technol., 59, 41 (2013).

[3] A. Nainani, T. Irisawa, Z. Yuan, B.R. Bennett, J. Brad Boos, Y. Nishi, K.C. Saraswat. IEEE Trans. Electron Dev., 58, 3407 (2011).

[4] Ch. Sun, M.T. Wade, Y. Lee, J.S. Orcutt, L. Alloatti, M.S. Georgas, A.S. Waterman, J.M. Shainline, R.R. Avizienis, S. Lin, B.R. Moss, R. Kumar, F. Pavanello, A.H. Atabaki, H.M. Cook, A.J. Ou, J.C. Leu, Y.H. Chen, K. Asanović, R.J. Ram, M.A. Popović, V.M. Stojanović. Nature, 528 (7583), 534 (2015).

[5] Ю.Б. Болховитянов, О.П. Пчеляков. УФН, $178(5), 459$ (2008).

[6] S.F. Fang, K. Adomi, S. Iyer, H. Morkoc, H. Zabel, C. Choi, N. Otsuka. J. Appl. Phys., 68 (7), R31 (1990).

[7] M. Akiyama, Y. Kawarada, K. Kaminishi. Jpn. J. Appl. Phys., 23 (11A), L843 (1984).

[8] T. Sakamoto, G. Hashiguchi. Jpn. J. Appl. Phys., 25 (1A), L78 (1986).

[9] D.J. Chadi. Phys. Rev. Lett., 59 (15), 1691 (1987).

[10] Е.А. Емельянов, Д.Ф. Феклин, М.А. Путято, Б.Р. Семягин, А.К. Гутаковский, В.А. Селезнев, А.П. Василенко, Д.С. Абрамкин, О.П. Пчеляков, В.В. Преображенский, N. Zhicuan, N. Наiqiao. Автометрия, 50 (3), 13 (2014).

[11] R.D. Bringans, D.K. Biegelsen, L.E. Swartz. Phys. Rev. B, 44 (7), 3054 (1991).

[12] M. Calamiotou, N. Chrysanthakopoulos, C. Lioutas, K. Tsagaraki, A. Georgakilas. J. Cryst. Growth, 227, 98 (2001).

[13] M.A. Putyato, V.V. Preobrazhenskii, B.R. Semyagin, D.F. Fëklin, N.A. Pakhanov, E.A. Emelianov, S.I. Chikichev. Semicond. Sci. Technol., 24 (5), 055014 (2009).

[14] K. Akahane, N. Yamamoto, S.I. Gozu, A. Ueta, N. Ohtani. J. Cryst. Growth, 283 (3-4), 297 (2005).

[15] S.H. Vajargah, S. Ghanad-Tavakoli, J.S. Preston, R.N. Kleiman, G.A. Botton. J. Appl. Phys., 114 (11), 113101 (2013).

[16] Y.H. Kim, Y.K. Noh, M.D. Kim, J.E. Oh, K.S. Chung. Thin Sol. Films, 518 (8), 2280 (2010).

[17] U. Serincan, B. Arpapay. Semicond. Sci. Technol., 34 (3), 035013 (2019).

[18] D.D. Firsov, O.S. Komkov, V.A. Solov'ev, P.S. Kop'ev, S.V. Ivanov. J. Phys. D: Appl. Phys., 49 (28), 285108 (2016).

[19] B. Méndez, P.S. Dutta, J. Piqueras, E. Dieguez. Appl. Phys. Lett., 67 (18), 2648 (1995)

[20] C.G. Van de Walle. Phys. Rev. B, 39 (3), 1871 (1989).

[21] M. Akiyama, Y. Kawarada, K. Kaminishi. Jpn. J. Appl. Phys., 23 (11A), L843 (1984).

[22] A. Georgakilas, J. Stoemenos, K. Tsagaraki, P. Komninou, N. Flevaris, P. Panayotatos, A. Christou. J. Mater. Res., 8 (8), 1908 (1993).

[23] R.L. Schwoebel. J. Appl. Phys., 40 (2), 614 (1969).

[24] Z. Ding, D.W. Bullock, P.M. Thibado, V.P. LaBella, K. Mullen. Surf. Sci., 540 (2-3), 491 (2003). 
[25] K. Ohta, T. Kojima, T. Nakagawa. J. Cryst. Growth, 95 (1-4), 71 (1989).

[26] T. Shitara, D.D. Vvedensky, M.R. Wilby, J. Zhang, J.H. Neave, B.A. Joyce. Phys. Rev. B, 46 (11), 6825 (1992).

Редактор Г.А. Оганесян

\title{
Influence of the crystallographic orientation of GaSb films on their structural properties during heteroepitaxy on vicinal $\mathrm{Si}(001)$ substrates by MBE
}

M.O. Petrushkov' ${ }^{1}$, D.S. Abramkin ${ }^{1,2}$, E.A. Emelyanov ${ }^{1}$, M.A. Putyato ${ }^{1}$, A.V. Vasev' ${ }^{1}$, D.I. Loshkarev', M.Yu. Yesin ${ }^{1}$, O.S. Komkov ${ }^{3}$, D.D. Firsov ${ }^{3}$, V.V. Preobrazhenskii ${ }^{1}$

${ }^{1}$ Rzhanov Institute of Semiconductor Physics, Siberian Branch of Russian Academy of Sciences, 630090 Novosibirsk, Russia

2 Novosibirsk State University, 630090 Novosibirsk, Russia

${ }^{3}$ St. Petersburg State Electrotechnical University "LETI", 197376 St. Petersburg, Russia

\begin{abstract}
GaSb films were grown by molecular beam epitaxy using $\mathrm{AlSb} / \mathrm{As} / \mathrm{Si}$ transition layers on vicinal $\mathrm{Si}(001)$ substrates deflected $6^{\circ}$ to the (111) plane. The influence of the $\mathrm{GaSb}$ films crystallographic orientation on their structural properties and surface morphology is investigated. It was found that $\mathrm{GaSb}(00 \overline{1}) / \mathrm{Si}$ films are characterized by better structural perfection, lower concentration of point defects and more planar and isotropic surface topography, compared with $\mathrm{GaSb}(001)$ films. Possible reason for the observed differences between $\mathrm{GaSb}$ films with different orientations is increased density of antiphase domains in $\mathrm{GaSb}(001)$ films. The morphological features of the grown films are caused by the terraces edges basically and by the anisotropy of the incorporation of $\mathrm{Ga}$ adatoms into the terraces edges, to a lesser extent.
\end{abstract}

